\title{
Biologically-inspired algorithms for object recognition
}

\section{Conference Paper}

Author(s):

Ternovskiy, Igor V.; Nakazawa, Dante; Campbell, Shannon; Suri, Roland Erwin (i)

Publication date:

2003

Permanent link:

https://doi.org/10.3929/ethz-a-007587129

Rights / license:

In Copyright - Non-Commercial Use Permitted 


\title{
BIOLOGICALLY-INSPIRED ALGORITHMS FOR OBJECT RECOGNITION
}

\author{
Igor Ternovskiy, Dante Nakazawa, Shannon Campbell*, Roland E. Suri \\ Intelligent Optical Systems \\ 2520 W. $237^{\text {th }}$ St., Torrance, CA 90505 \\ (310) $530-7130$ \\ correspondence to rsuri@intopsys.com
}

\begin{abstract}
Neurobiologically-inspired algorithms were investigated for their ability to recognize airplanes on satellite images. Standard segmentation algorithms often segregate objects in several segments or merge sections of objects to one segment. To correct such segmentation errors, we used information on the template object (topdown information) to correct such segmentation errors by merging several segments to one object (binding). This strategy was influenced by neurobiological findings demonstrating that top-down information modulates visual representations in primary visual areas. Our algorithms use gray-scale, size, moment of inertia, and distance between segments to detect segment groups that resemble airplanes. For the images that contained our template, 3 out of 3 large airplanes were correctly identified without false positives. Note that this successful identification was achieved despite the fact that in the original segmentation, each of the three recognized planes was broken up in several segments. In further simulations, we used the same template for 15 more airport images and achieved 8 correct matches, 29 missed matches, and 8 false positives. These results demonstrate that template information is indeed crucial to improve segmentation errors and can lead to substantial improvements of recognition rates. Further research should be conducted to systematically investigate the optimal criteria for reliable object detection.
\end{abstract}

\section{OVERVIEW}

The image recognition system derived area and contour information from a user-defined template and compared this template information with area and contour information in the image (Figure 1). A large airplane was defined by the user as the template by selecting and storing its three airplane segments. The algorithms found the three additional large airplanes without any false positives. Two smaller airplanes were not detected because our algorithms use size criteria. Note that although all the airplanes were segmented in at least two segments, the algorithms find the correct segment combinations that correspond to airplanes.
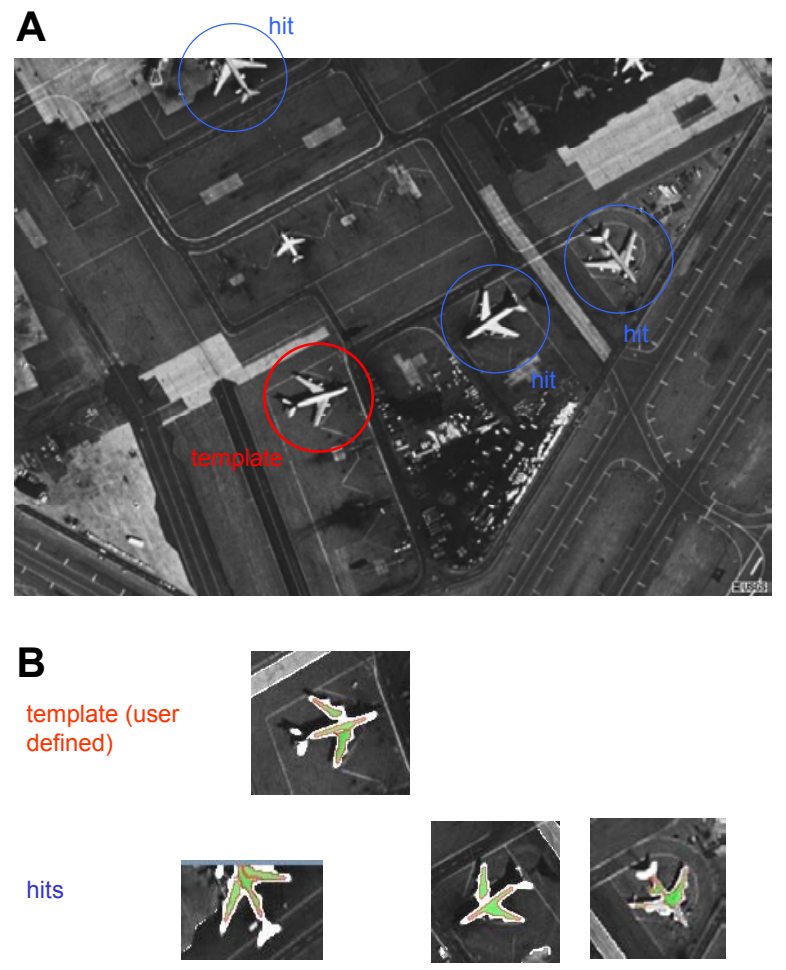

Figure 1. Airport image with search results: (A) The template airplane (red bold circle) was defined by the user. The algorithms detected three airplanes of similar size (blue circles); (B) Segmentation for template (top, 3 segments) and hits (bottom, 3, 2, and 2 segments).

An overview of the system is given in Figure 2. First, the user defines a template on an image for the object of interest. Second, traditional image processing steps are applied to derive useful features for the template image and a test image. Third, the algorithms search for matches between the template features and features detected on the test image. 


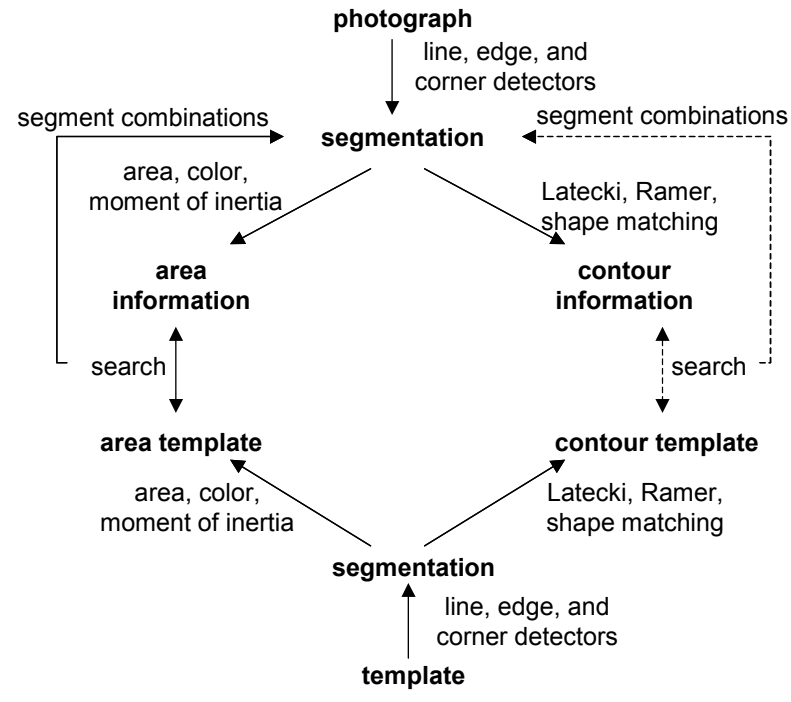

Figure 2. Overview of developed image recognition framework. A series of algorithms were developed including a SUSAN filter, and multiscale edge and line detection for the segmentation of the image (top). From the segmented image, area information such as gray-scale was computed for each segment. Combinations of segments were formed for the segments resembling airplanes in a first coarse search. For such segment combinations, the moment of inertia, distance between segments, and area size were computed (left). The algorithms proposed by Latecki (1972) and Ramer and collegues $(2002$ a,b) were used to approximate the segment borders with straight lines, which were required to compute contour information and segment angles (right, dashed lines not used for the shown results). The template was defined by the user as a typical example of the object (bottom). The template was processed with the same algorithms as the image (segmentation, area information, contour information). The features computed for the image were then compared with the features of the template using hierarchical search algorithms.

\section{SEgmentation Results}

Figure 3 (b) and (c) display segmentations of an example image by thresholding and region growing, respectively. In both segmentations, airplanes usually consisted of several segments.

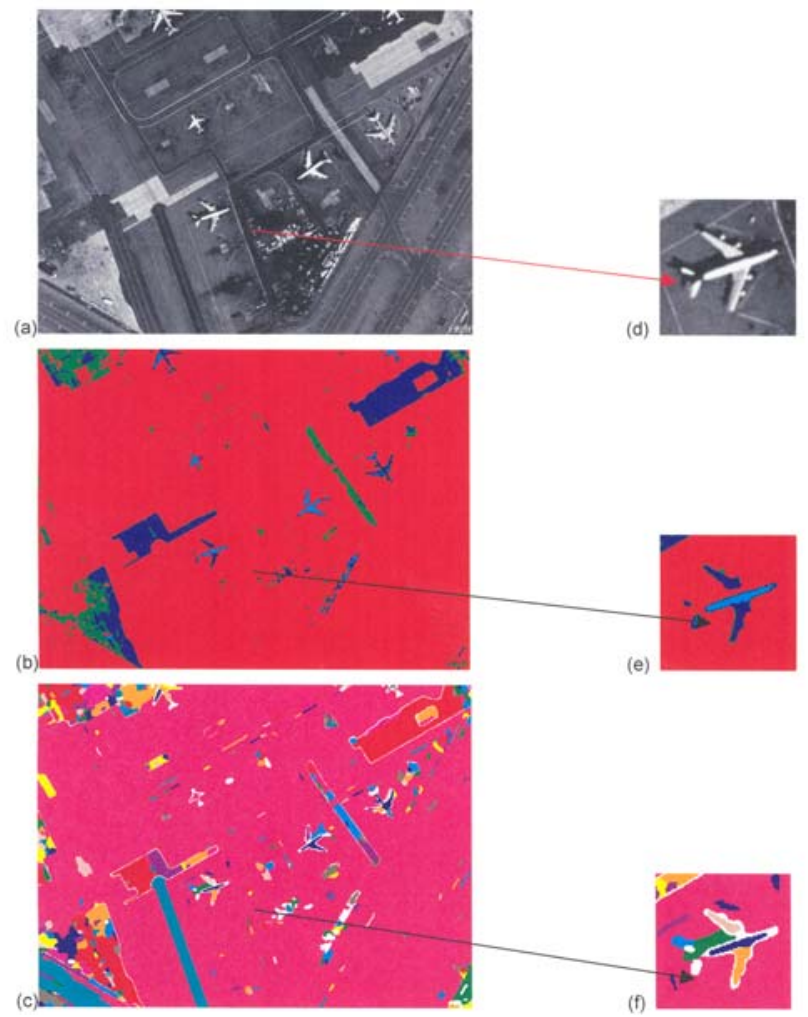

Figure 3. Satellite image of JFK airport. The images include: (a) the original grayscale, (b) the threshold segmentation of (a), (c) the region growing segmentation of (a), and the magnified section of the templates (d,e,f respectively) used in each of the images. For the images (b), (c), (e), and (f), each segment is colored in a single color to allow visual distinction of the segments. Colors were selected by chance for visual purposes and are irrelevant for the algorithms, as the segments were unequivocally labeled by the algorithms.

\section{IDENTIFICATION RELIABILITY}

The developed algorithms were tested on sixteen grayscale images of airports (Figure 4). We used the template of Figure 1 and Figure 3 for 15 more airports images and achieved 8 correct matches, 29 missed matches, and 8 false positives. These results strongly depended on the parameter settings, and by optimizing these parameters and/or by selecting more appropriate features, it should be possible to improve the object detection reliability. 

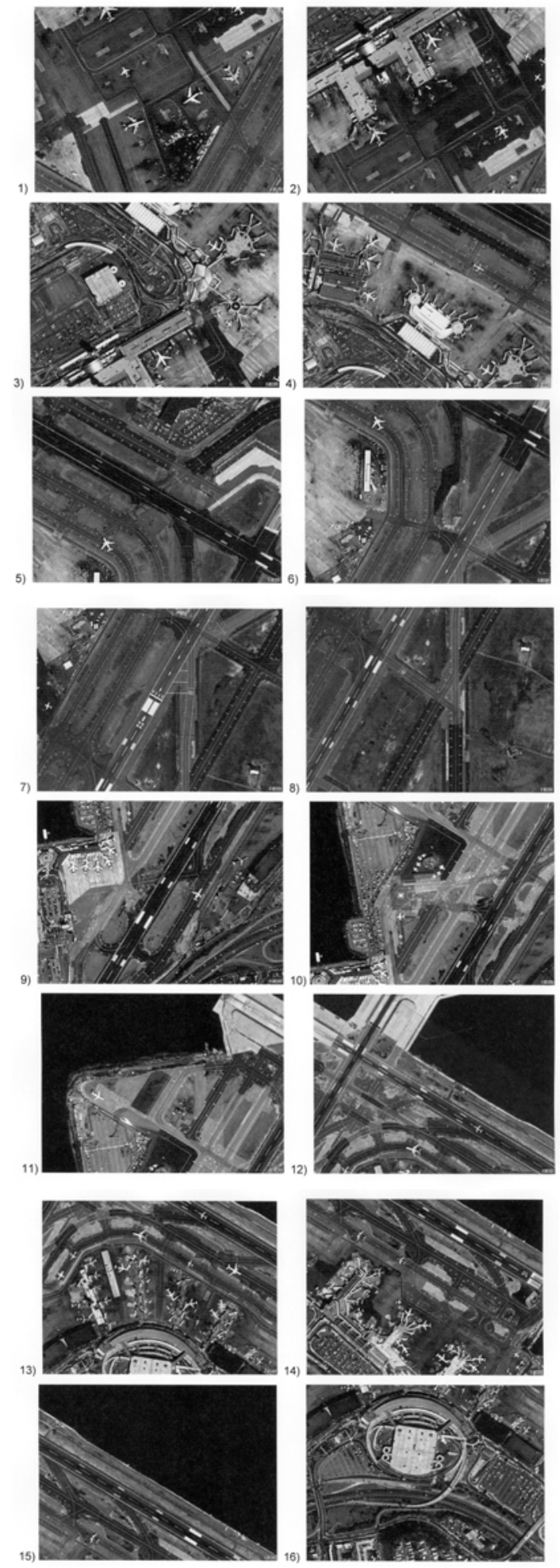

Figure 4. The sixteen test images of airports.

\section{Conclusions}

\section{Successes}

- Ease of use due to use of templates and graphical interface;

- Requires only one example object designed by the user to find similar objects;
- Fast - only about $10 \mathrm{sec}$ computing time per image; and

- Satisfactory performance without tuning of parameters.

\section{Problems}

- Since we are not aware of a benchmark data set, we were not able to compare the performance of our algorithms with that of competing algorithms.

- Angle information turned out to be unreliable, as the segmentations were not accurate enough to access angles (Figure 5). For this reason, angle information was not used for the simulation shown here. The accuracy problem may be solvable by using edge detectors to compute the angles, instead of combining segmentation algorithms proposed by Ramer (1972) or Latecki and collaborators (2002 a,b). Such edge detectors would correlate the gray-scale values of an edge template with all locations on the image, which is computationally expensive. This solution would be consistent with neurobiology, as there are local edge detector neurons in primary visual areas.

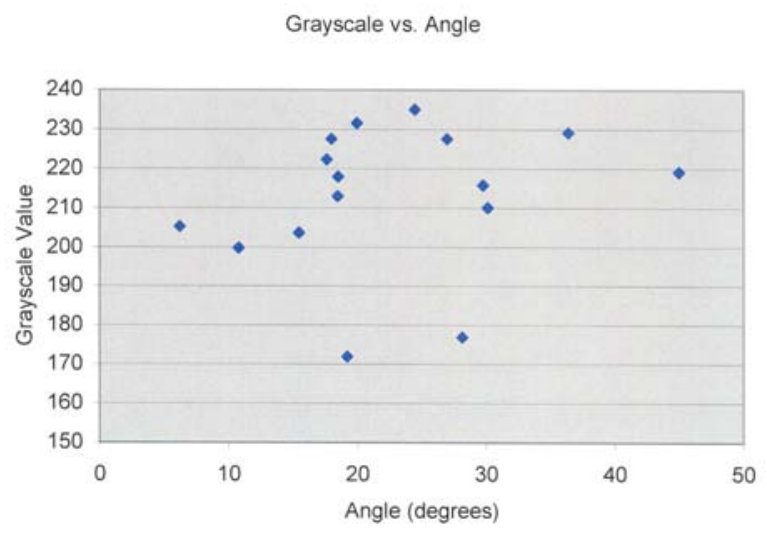

Figure 5. Graph of segment's grayscale value vs. wing tip angles of different planes. Although wing tips are salient for human observers, algorithms by Ramer and Latecki did not lead to sufficient accuracy of the wing tip angles. This lack of accuracy was not correlated with the gray scale value of the segment.

\section{REFERENCES}

L.J. Latecki and R. Lakamper, "Application of planar shape comparison to object retrieval in image databases," Pattern Rec., Vol. 35, pp. 15-29, 2002.

\section{L.J. Latecki, R. Lakamper, U. Eckhardt, Shape} Representation Project, www.math.unihamburg.de/projekte/shape/accessec, March 2002.

U. Ramer, "An iterative procedure for the polygonal approximation of plane curves," Comput. Graph. Imag. Proc. 1, pp. 244-256, 1972. 